\title{
Spot migration on the eclipsing binary KIC 9821078
}

\author{
E. Bahar, İ. Özavcı and H.V. Şenavcı \\ Department of Astronomy and Space Sciences, Faculty of Science, Ankara \\ University, Tandoğan 06100 Ankara, Turkey, (E-mail: \\ engin.bahar@ankara.edu.tr)
}

Received: October 31, 2019; Accepted: January 20, 2020

\begin{abstract}
High precision and continuous light curves obtained from the Kepler Space Telescope provide significant information about the behavior of cool starspots. In this study, we obtain surface maps of the eclipsing binary KIC 9821078 with the help of the light curve inversion method to reveal longitudinal spot migration from Kepler long-cadence (LC) light curves. We also present an upto-date solution from light and radial velocity curves. The inversion results find at least two dominant spot regions that migrate from lower to higher longitudes as a consequence of solar-like differential rotation.
\end{abstract}

Key words: Binaries: eclipsing - stars: magnetic activity - methods: light curve inversion - starspots: migration

\section{Introduction}

As we know from the Sun, stellar magnetic activity is one of the most important phenomena that affect stellar evolution as well as planet formation, although there are still unclear parts. Some methods, such as light curve inversion, give significant information about the underlying physics of stellar magnetic activity, using time-series light curves. Space telescopes (e.g. Kepler and TESS) offer unique opportunities to understand that phenomenon, with the help of high-precision, almost continuous data, covering long time intervals. We investigate longitudinal star-spot behavior and differential rotation of KIC 9821078 via light curve inversion (hereafter LCI) and frequency analysis (Lomb-Scargle periodogram) of high-precision and continuous Kepler light curves. We also derived up-to-date physical parameters.

KIC 9821078 is an Algol-type eclipsing binary with an orbital period of $\approx$ 8.43 days, in which the components are late type (K5/M0) (Pourbaix et al., 2004) low mass stars. The first detailed study of the system was by Devor et al. (2008). They mentioned that the component masses are consistent with current mass-radius models. They also derived physical properties from ground-based radial velocity and light curve data. Han et al. (2019) investigated cool spots on the components and pointed out that they are occulted during primary and secondary eclipses. They also argued that the components are synchronized with 
spots evolving over time, by examining residuals from a best-fit model. The system was described by Lurie et al. (2017) as having star-spot modulations. They measured multiple rotation periods with a combination of auto-correlation and the Lomb-Scargle periodogram.

\section{Observations and data reduction}

There are 17 quarters of long-cadence (hereafter LC) and 3 quarters of shortcadence Kepler light curve data for KIC 9821078. To eliminate brightness variations caused by instrumental or systematic influences, we reprocessed the photometry using the Python PyKE task (for more details see Özavcı et al. (2018)). In this work, we used only LC light curves, since they have sufficient data points in each cycle to perform LCI.

\section{System parameters and spot modelling}

To apply LCI we need parameters such as radii, mass ratio, and inclination. Therefore, we performed simultaneous analysis of Kepler light curves and radial velocity curve data from Han et al. (2019) to derive system parameters using PHOEBE software (Prša \& Zwitter, 2005). Derived parameters are highly compatible with those by Devor et al. (2008) and Han et al. (2019) and are listed in Tab. 1.

Table 1. Results from the light curve analysis of KIC 9821078

\begin{tabular}{cccc}
\hline \hline Stellar Parameters & Value & Absolute Parameters & Value \\
\hline$T 1(\mathrm{~K})$ & 4300 & $M_{1}\left(M_{\odot}\right)$ & $0.697(3)$ \\
$T 2(\mathrm{~K})$ & $3500(3)$ & $M_{2}\left(M_{\odot}\right)$ & $0.539(3)$ \\
$i\left({ }^{\circ}\right)$ & $88.97(1)$ & $R_{1}\left(R_{\odot}\right)$ & $0.642(2)$ \\
$a\left(R_{\odot}\right)$ & $18.69(2)$ & $R_{2}\left(R_{\odot}\right)$ & $0.552(1)$ \\
$q$ & $0.7734(16)$ & $L_{1}\left(L_{\odot}\right)$ & $0.142(1)$ \\
$V_{\gamma}(\mathrm{km} / \mathrm{s})$ & $-24.02(5)$ & $L_{2}\left(L_{\odot}\right)$ & $0.041(1)$ \\
$e$ & $0.00070(4)$ & $\log g_{1}[\mathrm{cgs}]$ & $4.666(1)$ \\
$\ldots$ & $\ldots$ & $\log g_{2}[\mathrm{cgs}]$ & $4.687(1)$ \\
\hline \hline
\end{tabular}

LCI is one of the techniques commonly used to obtain starspot-induced brightness distributions of star surfaces from light curves. We first phase the light curves into chunks. If the system is synchronized, as assumed by Devor et al. (2008) and Han et al. (2019), then we can use the orbital period for phasing. However, using auto-correlation and Lomb-Scargle periodograms, Lurie et al. (2017) found that the rotational and orbital periods differ significantly. In this 
context, we computed the generalized Lomb-Scargle periodogram from out-ofeclipse Kepler LC light curves and found two strong peaks that correspond to 9.775 and 10.113 days, similar to those estimated by Lurie et al. (2017). The light curves and the periodogram are in Fig. 1(a). Those periods can be related to component rotations and/or spot migration. Those two periods as well as the orbital period were applied for the cycle chunk phasing.

We used the DoTS code (Collier Cameron, 1997) to perform LCI. We first used the orbital period ( $P_{\text {orb }}=8.429$ days) to reconstruct the surface map for each cycle. Afterwards, we obtained a measure of the relative spot filling factor as a function of longitude and examined how starspots behave as a function of time, throughout $\approx 4 \mathrm{yr}$. This process was performed for two other periods mentioned above. The resultant starspot patterns for three periods are in Fig. 2.

\section{Results and discussion}

We have presented a detailed investigation of activity variations and differential rotation in the K5 type primary component. We applied the LCI method assuming the spots to be on the primary component since, a) the primary has most of the light $(\approx 80 \%)$ and b) light curves, in general, have only limited information for identifying the spotted component. We obtained three longitudinal spot variations and corresponding drift rates using periods 8.429, 9.775 and 10.113 days. It is not possible to measure directly the drift rate(s), since the periods determined from frequency analysis include not only the rotation periods but the migration rates. It is clear from Fig. 2 that period differences affect only the drift rates (slopes), while it is still possible to infer the drift directions and estimate spot latitudes with $\approx 4$ years of time-series data. This is best visible in Fig. 2(b) that gives clues on the presence of at least two spot regions that migrate toward increasing longitudes with different drift rates between cycles 60 and 80. Furthermore, spot regions that are formed and disappeared in different longitudes can also be clearly seen over time. We also find two magnetic activity cycles of about $0.78 \mathrm{yr}$ and $2.02 \mathrm{yr}$ with Lomb-Scargle peridograms for variations of total spot coverage fraction (see Fig. 1(b)).

We also determined the amount of surface shear, as computed from the periods determined with the generalized Lomb-Scargle periodogram in Fig. 1(a). More details on the determination of surface shear can be found in Reinhold $\&$ Arlt (2015). We obtained the amount of surface shear as $\alpha \cong 0.033$, which indicates the existence of solar-like differential rotation.

Time-series high-resolution spectra are needed to better understand the system's activity.

Acknowledgements. The authors acknowledge the support by The Scientific And Technological Research Council Of Turkey (TÜBITAK) through the project 1001 $115 \mathrm{~F} 033$. 

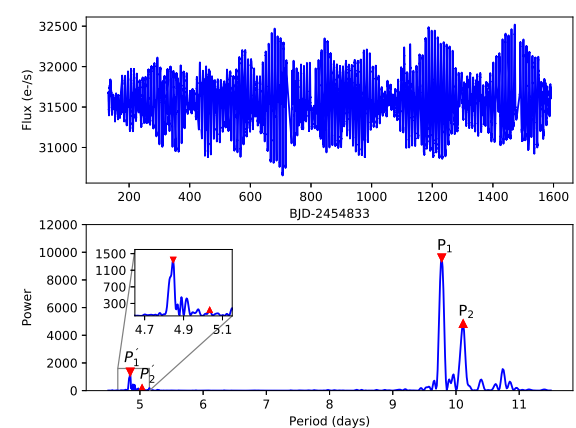

(a)

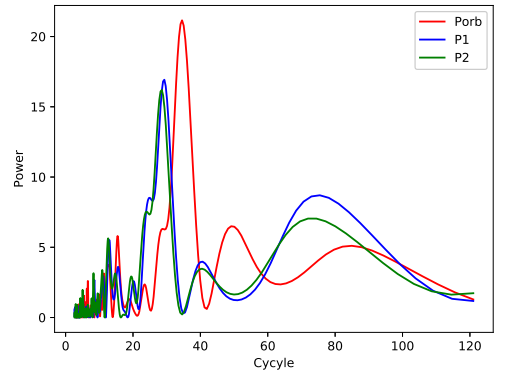

(b)

Figure 1. (a-top): Full Kepler light curves of the system without eclipses and generalized Lomb-Scargle periodogram for the light curves. $P_{1}$ and $P_{2}$ show two strong peaks in the diagram at 9.775 and 10.112 days, respectively, while $P_{1}^{\prime}$ and $P_{2}^{\prime}$ are their first harmonics (a-bottom). (b): Lomb-Scargle periodogram for variations of total spot coverage fraction over time.

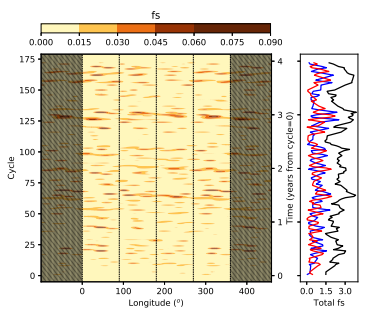

(a)

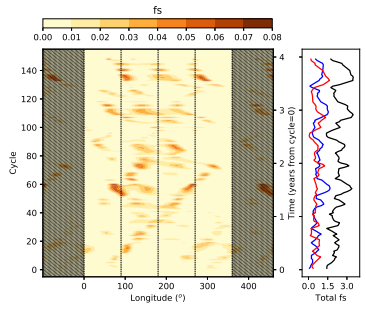

(b)

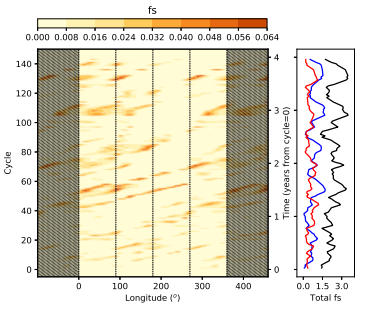

(c)

Figure 2. KIC9821078 time-longitude diagrams for the periods 8.429 days (a), 9.775 days (b), and 10.112 days (c). The right panels of each diagram show variations of total (black line) and two sets of opposite longitudinal hemispheres spot coverage fraction over time.

\section{References}

Collier Cameron, A., Eclipse mapping of late-type close binary stars. 1997, Mon. Not. R. Astron. Soc., 287, 556, DOI: 10.1093/mnras/287.3.556

Devor, J., Charbonneau, D., Torres, G., et al., T-Lyr1-17236: A Long-Period Low-Mass Eclipsing Binary. 2008, Astrophys. J., 687, 1253, DOI: 10.1086/592080

Han, E., Muirhead, P. S., \& Swift, J. J., Magnetic Inflation and Stellar Mass. IV. Four Low-mass Kepler Eclipsing Binaries Consistent with Non-magnetic Stellar Evolutionary Models. 2019, Astron. J., 158, 111, DOI: 10.3847/1538-3881/ab2ed7 
Lurie, J. C., Vyhmeister, K., Hawley, S. L., et al., Tidal Synchronization and Differential Rotation of Kepler Eclipsing Binaries. 2017, Astron. J., 154, 250, DOI: 10.3847/1538-3881/aa974d

Özavcı, I., Şenavcı, H. V., Işık, E., et al., Recurrent star-spot activity and differential rotation in KIC 11560447. 2018, Mon. Not. R. Astron. Soc., 474, 5534, DOI: 10.1093/mnras/stx3053

Pourbaix, D., Tokovinin, A. A., Batten, A. H., et al., The ninth catalogue of spectroscopic binary orbits. 2004, Astron. Astrophys., 424, 727, DOI: 10.1051/00046361:20041213

Prša, A. \& Zwitter, T., A Computational Guide to Physics of Eclipsing Binaries. I. Demonstrations and Perspectives. 2005, Astrophys. J., 628, 426, DOI: 10.1086/430591

Reinhold, T. \& Arlt, R., Discriminating solar and antisolar differential rotation in high-precision light curves. 2015, Astron. Astrophys., 576, A15, DOI: 10.1051/00046361/201425337 